\title{
Epigenetic control of signaling networks involved in interleukin-18-induced cardiac hypertrophy and its attenuation by pan-histone deacetylase inhibitors
}

\author{
Gipsy Majumdar ${ }^{1 *}$, Rajendra Raghow ${ }^{1,2}$ \\ From UT-ORNL-KBRIN Bioinformatics Summit 2010 \\ Cadiz, KY, USA. 19-21 March 2010
}

\section{Background}

Daily intra-peritoneal injection of IL-18 for one week in $\mathrm{Balb} / \mathrm{c}$ mice led to the development of severe cardiac hypertrophy that was accompanied by induction of fetal gene expression. Both anatomical and molecular features of cardiac hypertrophy were ameliorated by pan-histone deacetylase inhibitors (HDACIs), m-carboxycinnamic acid bis-hydroxamide (CBHA) and trichostatin A (TSA).

\section{Materials and methods}

Treatment of mice with IL-18, with or without CBHA or TSA, caused dynamic remodeling of cardiac chromatin as judged by altered posttranslational modifications of the constituent histones. Administration of either TSA or CBHA in mice, even in the presence of IL-18, induced hyper-acetylation of histone $\mathrm{H} 3$ at lysine 9 demonstrating the unique action of HDAC inhibitors. Epigenetic changes in chromatin architecture were accompanied by reprogramming of cardiac hypertrophy-specific gene expression. Comparative profiles of cardiac gene expression of vehicle-treated mice with animals that received IL-18 \pm CBHA or TSA revealed that 184 genes were differentially expressed genes (DEGs) and a subset of 38 genes was oppositely regulated by IL-18 and TSA. In contrast, the hearts of mice treated with IL-18 and/or CBHA elicited 147 DEGs; IL-18 robustly up-regulated 58 genes that were blocked by CBHA or IL-18+CBHA.

\section{Results and conclusion}

Ingenuity Pathway Analysis of DEGs revealed that both HDACIs profoundly impacted a number of intracellular H9c2 cardiac myocytes incubated with CBHA or TSA for 6 and $24 \mathrm{hr}$ were also carried out. We observed that the signatures of DEGs evoked by TSA and CBHA after 6 and at $24 \mathrm{~h}$ treatment were unique. Nevertheless, it appeared that both HDACIs altered the course of PI3KPTEN-Akt/PKB, $\mathrm{Ca}^{++} /$calmodulin and p38-ERK-MAPKJNK signaling pathways to regulate gene expression via activation of Ap1, HNF4A, Myc and NFkB. Informed by the putative signaling networks deduced from the DEGs seen in the heart, we investigated the expression of phosphatase and tensin homolog (PTEN), a key regulator of the PI3K-AKT/PKB-NFkB pathway. Both TSA and CBHA induced robust expression of PTEN mRNA and protein in the heart. Based on these data we conclude that both TSA and CBHA attenuated anatomical and functional manifestations of IL-18-induced cardiac hypertrophy by epigenetic induction of PTEN to selectively block the PI3K-AKT/PKB-NFkB signaling.

\section{Author details \\ 'Department of Veterans Affairs Medical Center, Memphis, TN 38104, USA. ${ }^{2}$ Department of Pharmacology, University of Tennessee Health Science Center, Memphis, TN 38163, USA.}

Published: 23 July 2010

\section{doi:10.1186/1471-2105-11-S4-P1}

Cite this article as: Majumdar and Raghow: Epigenetic control of signaling networks involved in interleukin-18-induced cardiac hypertrophy and its attenuation by pan-histone deacetylase inhibitors . BMC Bioinformatics 2010 11(Suppl 4):P1.

* Correspondence: gmajumdar@uthsc.edu

${ }^{1}$ Department of Veterans Affairs Medical Center, Memphis, TN 38104, USA 